\title{
Applying the Business Process and Practice Alignment Meta-model: Daily Practices and Process Modelling
}

\author{
Paula Ventura Martins, Marielba Zacarias \\ University of Algarve, Portugal
}

\section{Abstract}

Background: Business Process Modelling (BPM) is one of the most important phases of information system design. Business Process (BP) meta-models allow capturing informational and behavioural aspects of business processes. Unfortunately, standard BP meta-modelling approaches focus just on process description, providing different BP models. It is not possible to compare and identify related daily practices in order to improve BP models. This lack of information implies that further research in BP meta-models is needed to reflect the evolution/change in BP. Considering this limitation, this paper introduces a new BP meta-model designed by Business Process and Practice Alignment Meta-model (BPPAMeta-model). Our intention is to present a meta-model that addresses features related to the alignment between daily work practices and BP descriptions. Objectives: This paper intends to present a metamodel which is going to integrate daily work information into coherent and sound process definitions. Methods/Approach: The methodology employed in the research follows a design-science approach. Results: The results of the case study are related to the application of the proposed meta-model to align the specification of a BP model with work practices models. Conclusions: This meta-model can be used within the BPPAM methodology to specify or improve business processes models based on work practice descriptions.

Keywords: business process, daily practices, business process modelling, meta-model JEL classification: $\mathrm{O} 31$

Paper type: Research article

Received: Mar 31, 2016

Accepted: Dec 28, 2016

Citation: Martins, P.V., Zacarias, M. (2017), "Applying the Business Process and Practice Alignment Meta-model: Daily Practices and Process Modelling", Business Systems Research, Vol. 8, No. 1, pp. 1-16.

DOI: 10.1515/bsrj-2017-0001

\section{Introduction}

Business process modelling (BPM) specializes on describing how activities interact and relate with each other, and how activities interact with other business concepts such as goals and resources, where resources may be material and informational entities, as well as human or automated actors. BPM considers organizations as entities mainly driven by processes and process-related concepts such as activities, 
tasks, resources, decisions and workflows as the main perspective of an organization (Hollingaworth, 2004). However, process execution is affected by many factors not included in process models. Indeed, the enacted organization is composed by a complex and adaptive web of human and automated actors acting and interacting with each other. Interactions among actors are both supported and constrained by information systems and tools, shared vocabularies and meanings, interaction patterns and rules. Moreover, business process execution is in constant evolution and current BPM languages are not able to cope with such evolution (Castela et al., 2012).

BPM languages do address properly high-level process descriptions, because at that level, processes are generally fairly stable. Lower-level descriptions though are more difficult because they exhibit greater variability. In general, organizations are not able of fully-describing their process models due to lack of detailed information, and the tacit and decentralized nature of the knowledge required (Verner, 2004). The problem of process variability and resulting unpredictability is addressed by (Mutschler et al., 2008; Reichert et al., 2008). Research on agile BPM (Bider et al., 2016) aims at managing the evolving nature of processes by using principles and practices from the software engineering community. Yet, there is still little guidance regarding the problem of (1) tacit knowledge and (2) means for keeping an up-todate alignment between business process models and actual execution.

From our point of view, actual execution is better captured by work practices rather than procedures or business process specifications. The term work practice comes from socio-technical approaches to system analysis and design, organizational anthropology, and management studies (Sierhuis et al., 2000). Work practices not only capture action and interaction patterns with high levels of detail. The patterns reflect behaviours of specific individuals and groups over time, rather than generic and static behaviours expected from job roles. Furthermore, work practice reflect the particular circumstances or conditions in which given behaviours are exhibited, the usage of machines, tools, information sources and other artefacts. Consequently, modelling work practice provides a deeper understanding of the human and automated activities that compose business processes, and is better suited to capture changes that trigger business process transformations in time.

Considering the aspects described previously, the authors proposed a Business Process and Practice Alignment Methodology (BPPAM) (Zacarias et al., 2014) for business process improvement, which set out principles and strategies for improving quality of business processes, based on actual work practices. This methodology provides guidance about how knowledge about organizational practices is gathered to improve business processes improvement. A key driver of BPPAM, concerning business process improvement is the ability to facilitate the alignment of business processes improvement activities and daily work practices. In this regard, this paper focuses on a meta-model to integrate daily work information into coherent and sound process definitions.

The remainder of this paper is organized as follows: section 2 presents a brief analysis about business process meta-models and work practice modelling. Section 3 describes the methodology applied in this research work. In section 4 we present the proposed Business Process and Practice Meta-model (BPPAMeta-model). Section 5 reports preliminary results of exploring the usage of BPPAMeta-model. Finally, section 6 concludes and discusses future trends. 


\section{Literature Review}

The literature review is organized in three parts including the basic overview on business process meta-models, work practices modelling and limitations related with these approaches.

\section{Business Process Meta-Models}

This section describes several business process meta-models, a subject of several standardization efforts. These meta-models are a basis to the BPPAM methodology, briefly described in the introduction. These approaches comprise a set of concepts to capture several aspects of business processes. In particular, the business process meta-model allows capturing functional, informational and behavioural aspects of business processes. The following present an outline of three meta-models with the strengths and weakness of each approach to justify the creation of our meta-model proposal, we do not represent each meta-model in full detail.

Figure 1

BPMN Meta-metamodel

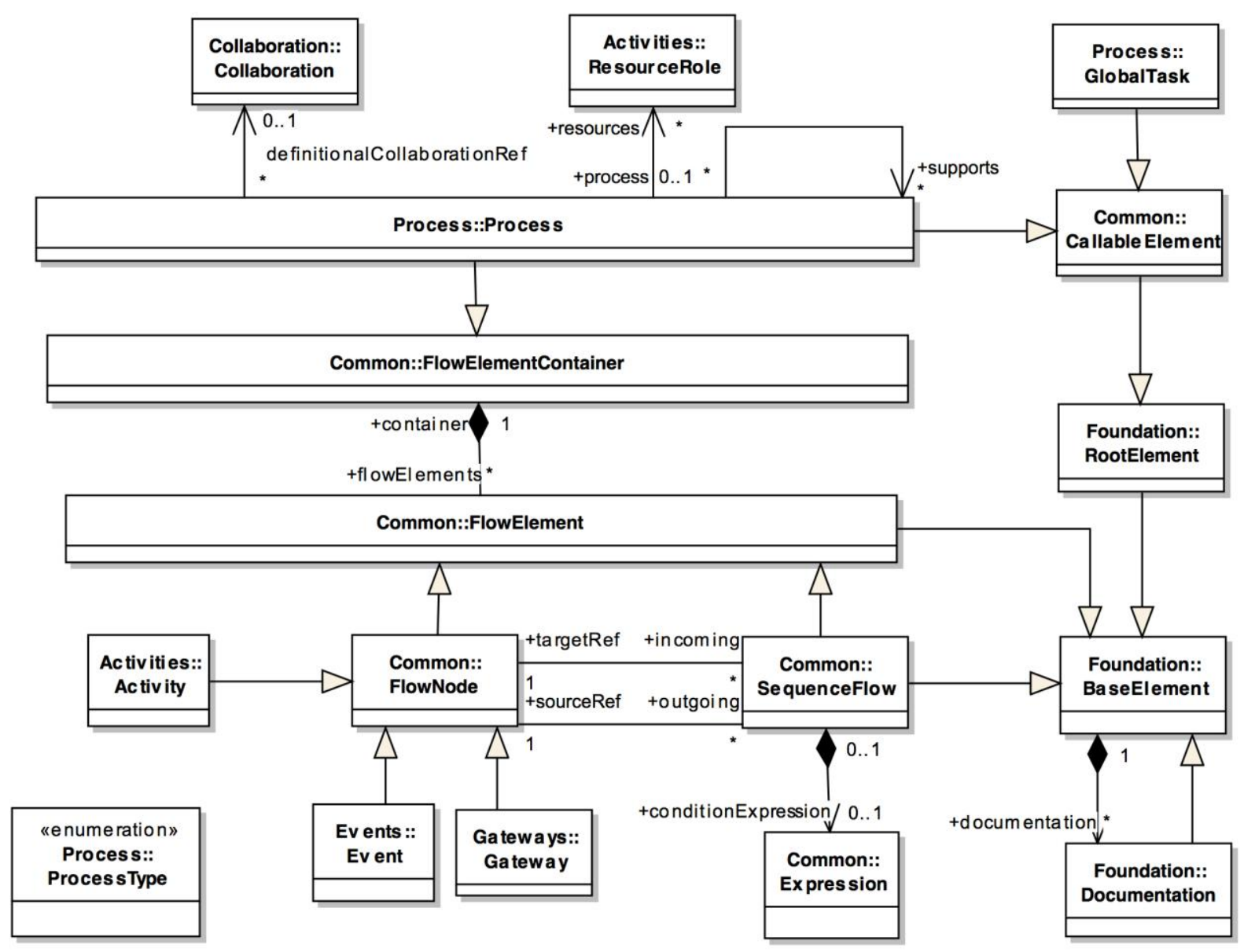

Source: Adapted from OMG (2013)

The Business Process Model and Notation (BPMN) Meta- model (OMG, 2013) was defined by the Object Management Group as a de facto standard that holds all definitions common to process oriented models. The BPMN is structured in several layers, the most important is the Core layer that contains 3 sub-packages: Foundation, a package with fundamental constructors for modelling; Service, a 
package that includes constructors for services and interfaces modelling; Common, a package with the classes that are common to the layers of Process, Choreography and Collaboration. Since our focus is business process metamodelling approaches, we only describe the Process meta-model and ignore the others because they are out of scope of this study.

Figure 2

Quality-Oriented Business Process Meta-model

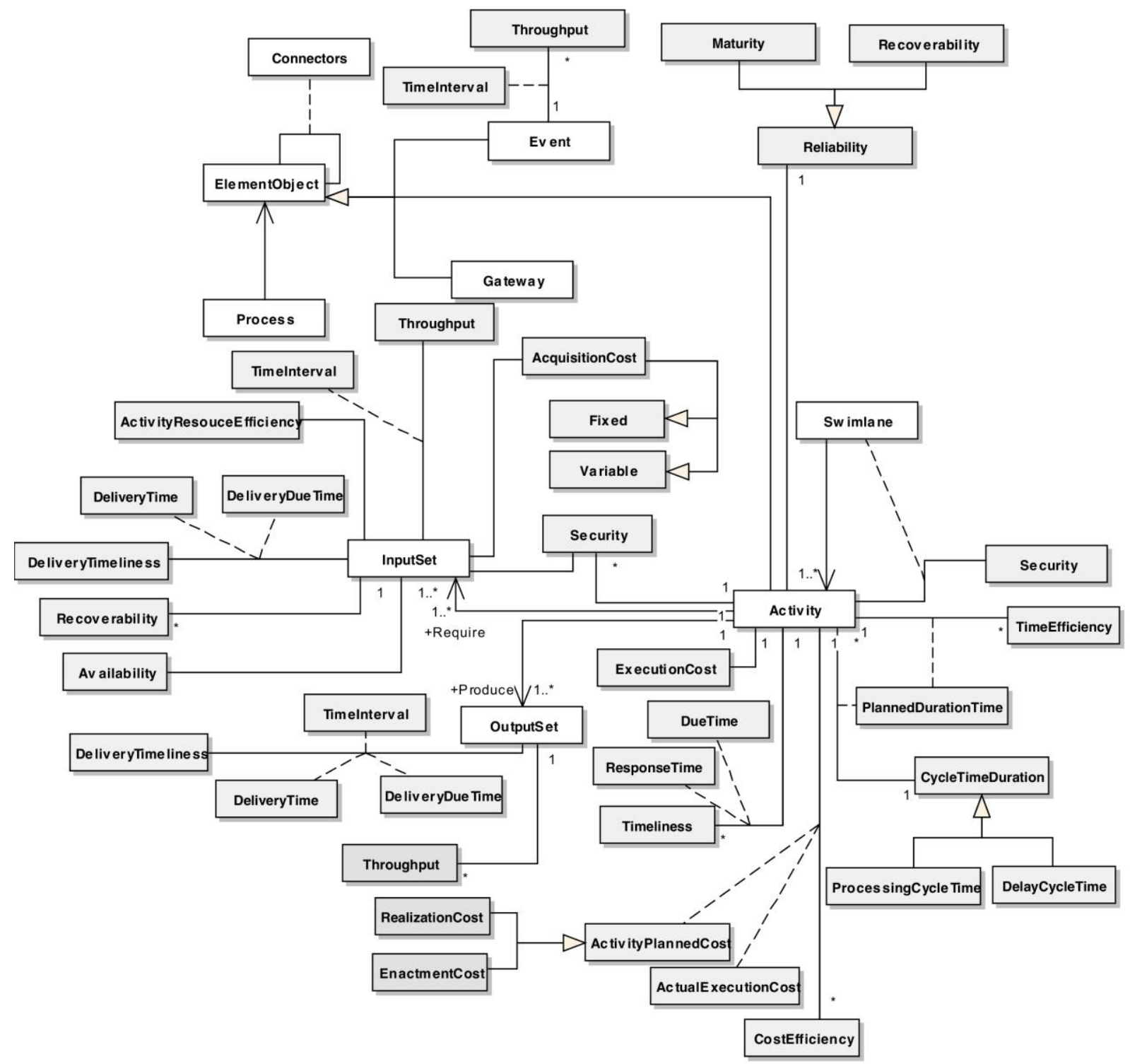

Source: Adapted from Heidari et al. (2011)

The meta-classes of the Process Meta-model are depicted in Figure 1; the illustration shows the term Collaboration used to model interactions between processes. A Process contains several FlowNodes (Activity, Event, Gateway) connected by SequenceFlows. A SequenceFlow shows the order in which activities are performed in a process, and relates activities, gateways and events to each other. A Process has several resources that will perform or will be responsible for that Process which are designed by ResourceRole. 
The Quality-Oriented Business Process Meta-Model (QOBPM) (Heidari et al., 2011) besides providing a unified view of all business process constructs and related quality dimensions also serves as a basis for business process quality evaluation. The main contribution of this approach was the assignment of quality information meta-classes to the corresponding business process constructs, which are grey-coloured in Figure 2. The different types of elements of a business process are: Activity, Event, Gateway and Connectors. This meta-model has been designed integrating the concepts existing in seven different business process modelling techniques (BPMN, IFED0, IFED3, RAD, UML-AS, SADT and EPC).

Figure 3

Transactional Meta-model Business Process (Business Process Package)

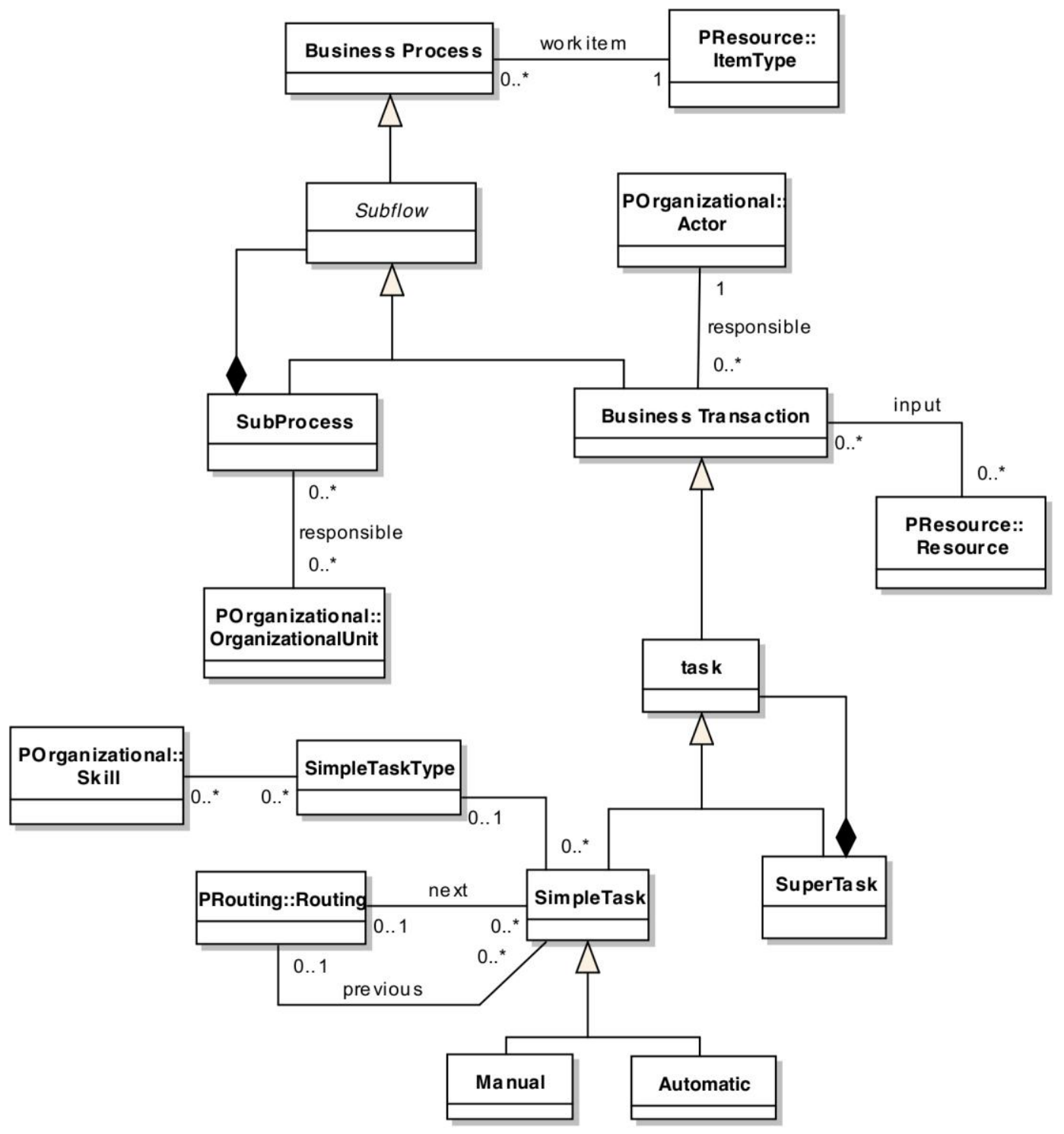

Source: Adapted from Thom et al. (2005)

The Transactional Meta-model for Business Process (TMBP) (Thom et al., 2005) is composed of five packages: Business Process, Organizational, Resource, Routing and Catalogue. The Organizational package differentiates between functional and organizational roles (Figure 3). The Resource package identifies different types of 
resources. The Routing package determines the order of tasks execution. The Catalogue package allows the selection of the best design pattern from a catalogue of business (sub-) process patterns to model certain business process. The Business Process package describes a business process which can involve several business transactions that can be decomposed into tasks. The relationship between the package meta-classes and the actor, resource, organizational unit, skill and routing meta-classes of the other packages are also included in the diagram.

\section{Work Practice Modelling}

In order to better comprehend what the concept of work practices entails, we must distinguish between procedures and practices. Procedures are specifications that define how tasks should be accomplished, and who is responsible for each task (Degani et al., 1997). Work practices reflect how people enact procedures.

Since different individuals and groups have different skills, habits, preferences, values and personalities, the degree to which they follow procedures is highly variable and thus deviate from procedures in varying measures. Fine-grained process descriptions, activity, and task models represent standard operating procedures, they are not able of representing actual work practices. Modeling work practices offers a means of uncovering problems not detected in process or tasks models. Some research efforts in work practice modelling include a context model and representation language developed by Pomerol and Brézillon (2011). A premise of this work is that the main distinction between operational procedures and practices is the context where these practices apply. Their model of context relates the notion of context and knowledge. At each moment, context is what surrounds a given focus of attention (e.g. a particular step of a task at hand). Proceduralized context is in fact part of contextual knowledge; however, it is put together and reorganized in order to solve a problem. The authors model context using acyclic graphs with two basic components; actions and contextual elements.

Sierhuis and Clancey (1997) developed a language called BRAHMS (Business Redesign Agent-based Holistic Modelling System). BRHAMS is part of a modelling environment based on agents and activities, where people are the center of the model instead of activities because their premise is that knowledge cannot be disembodied from them. BRAHMS capture what agents do throughout the day, not just the activities they perform. The language is focused on capturing knowledge and learning in human activities. It combines the perspective of business processes with a cognitive perspective to make social processes visible by capturing the knowledge that each agent has of other agents allowing a proper work distribution, seeking support from others and prioritizing jobs. Thus, Brahms not only models standard task flows but also how work get done, emphasizing practices, and individual productivity statistics. In this sense, BRAHMS enriches work-related concepts (activities and work frames i.e. rules that model situations that trigger actions), other concepts such as detectables (facts of the world, probability of occurrence during a particular action). Detectables may represent conditions that cause interruptions to workframes or ending them.

Zacarias et al. (2010) propose a model based on contexts and agents to capture and model work practice by representing agent behavior from three different perspectives, action, deliberation and learning/change. The action layer captures recurrent behavior using concepts such as actions, resources (information items, tools and human). These concepts are combined to represent action and interaction patterns. The model acknowledges the contextual nature of these 
patterns by associating them a particular context. The deliberation layer captures the rules used to activate or deactivate action or interaction contexts. The main concepts of this layer are context activation rules, interpersonal rules, to-do lists, events and commitments. In other words, this layer captures scheduling rules and multi-tasking behavior. Thereafter, the deliberation layer enables to see the practices used by people in managing themselves. The change/learn layer captures rules that constrain possible changes to the concepts in action and deliberation layers.

\section{Limitations in process and practice modelling}

Although, there is no current standard core business process meta-model, all the three meta-models presented use more or less the same concepts and don't support work practice modelling. As final conclusion, BPMN also integrates orchestration and choreography. The QOBPM considers all possible constructs of a business process but enriched with quality information to effectively assess the quality of business processes. Finally, the TMBP links organizational structure aspects with business (sub) process and makes it feasible the reuse of business (sub)process patterns to create business (sub)process.

Regarding current work practice modelling approaches, they are mostly informal. Hence, no formal meta-model has been proposed, as is the case for several business processes modelling approaches. Since current business process meta-model lack constructs for work practice modelling, no means are provided to address the alignment between business process and work practices.

\section{Methodology}

In 2004, (Hevner et al., 2004) proposes an approach to Information Systems research that combines behavioral and design science research. In this approach, the environment surrounds goals define business goals and business needs identified by members of the organization. Based on given business needs, behavioral science research develops and justifies theories explaining business phenomena. Within Information Systems research, such phenomena involve human actors, organizations and supporting technologies. Design-science builds and evaluates artifacts to satisfy business needs previously agreed upon. Whereas behavioral research aims at finding a given truth, the goal of design science research is utility of artifacts. An artifact maybe useful due to a still unknown truth. Incorporating a given truth into an artifact design requires developing theories, which are later assessed through evaluation and justification activities and lead to further refinements of the theory.

The methodology employed in the present research follows the design-science approach of Hevner and colleagues. As aforementioned, design science encompasses two complementary activities; building artifacts to meet specific business needs or solving a given problem, and evaluating the utility of the artifacts regarding the satisfaction of the respective needs or the problems intended to be solved. The artifacts built by design science include constructs, models, methods and instantiations. Constructs allow defining the language to build models, and define problems and solutions. Models provide means of exploring the effects of designs on the real world. Methods define ways of solving specific problems. Finally, instantiations show implementations of artefacts in working systems.

This paper describes an artefact, a meta-model to facilitate the alignment between business processes and work practices that was built as part of our research. The objective of the remaining research process is to evaluate the utility of 
meta-model in real business environments, regarding the particular needs identified within such environments.

\section{BPPAMeta-model}

Business Process and Practice Alignment Methodology (BPPAM) intends to establish disciplined business process practices based on daily actions. To support this approach, it is important to define and describe business processes and daily practices.

Figure 4

Business Process and Practice Alignment Meta-model

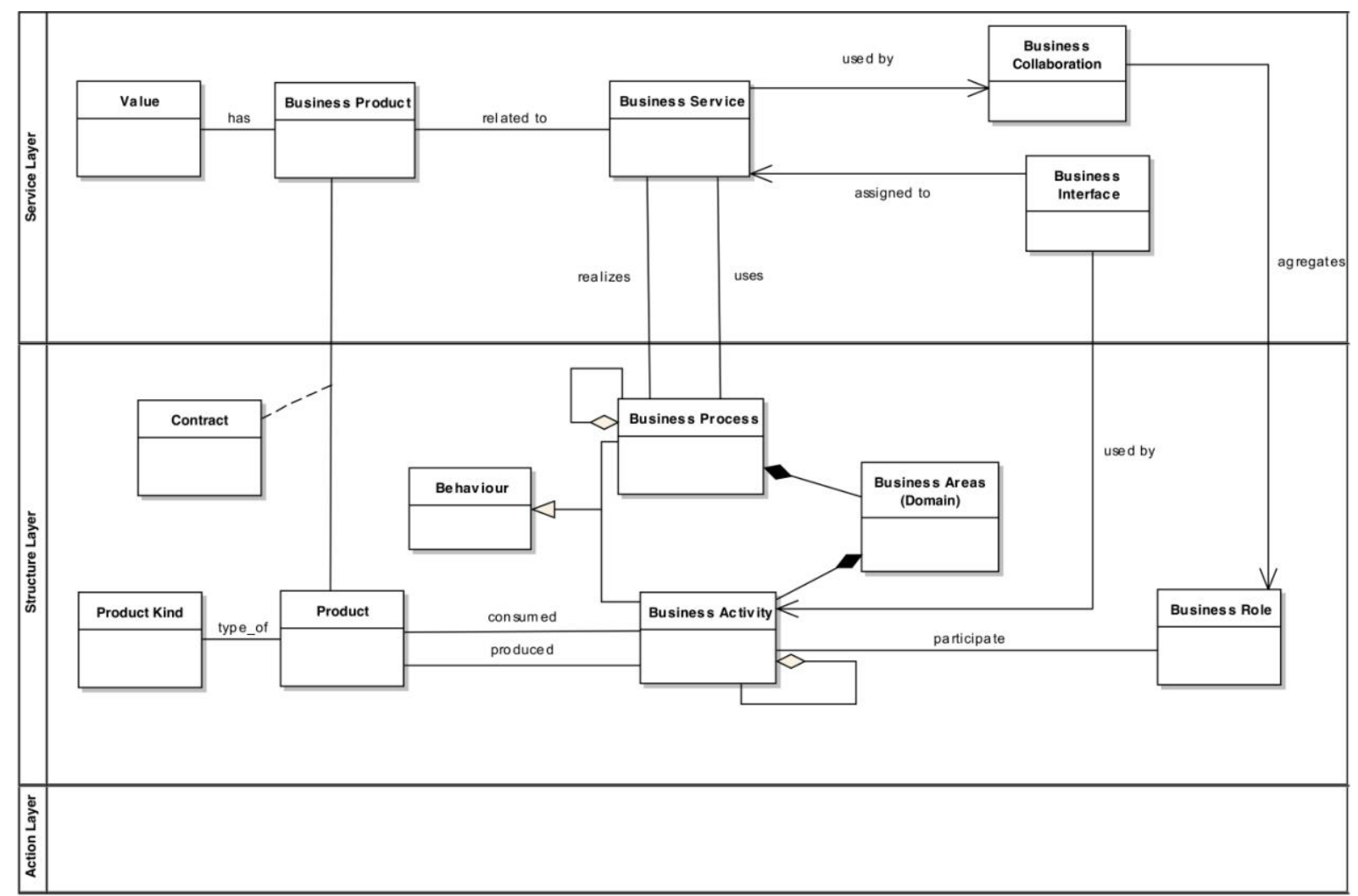

Source: Author's illustration

Business process modelling aims to describe the actually performed business process, the models are used as the basis for understanding and analysing processes, improving existing processes, as a baseline for process changes or for disseminating process knowledge. Nevertheless, existing meta-modelling approaches don't cover aspects related to daily actions and also do not solve the gap regarding how to use elements from daily practices to create business process elements. In order to provide support for these aspects, an extra layer is included in our meta-model. This extension also intends to describe the relation between business processes and daily practices. Figure 4 illustrates BPPAM meta-model that has three layers: service layer, structure layer and action layer. Each layer is focused on a specific set of concerns and encompasses several elements that describe the concerns of the layer. Considering the complexity of the action layer, the representation of its elements is showed in a separated figure (Figure 5). 
The service layer offers business products and business service to external costumers, which involves some business collaborations. The basic elements are:

- Business Service - unit of functionality that supports a business that hides internal activities.

- Business Product - goods that are sold to other businesses, and used to produce other goods.

- Business Value - satisfying the needs and expectations of the costumer.

- Business Collaboration - join effort of multiple work groups to accomplish a business service.

- Business Interface - point of access where a set of activities is made available to customers.

Figure 5

Action Layer of the BPPAMeta-model

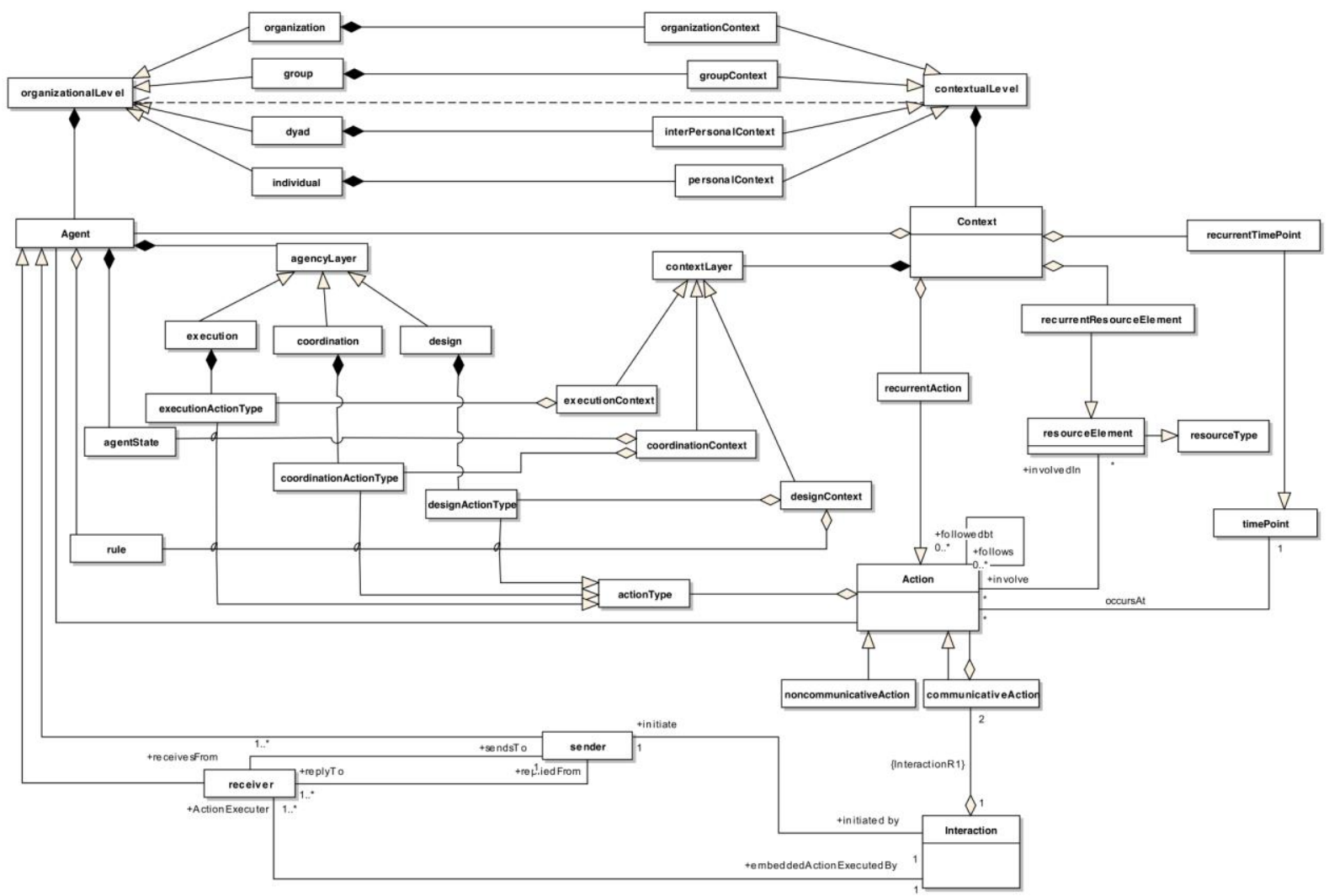

Source: Author's illustration

The structure layer of the BPPAM meta-model represents the elements (metaclasses) that are relevant for modelling the functional aspect (activity, process), informational aspect (product and product kind), behavioural aspects and organizational aspects (role and actor). These basic elements are:

- Behaviour - best practices that guide an organization.

- Business Process - is a behaviour element based on a set of ordered activities. It is intended to produce products or business services.

- Business Areas - an organizational unit corresponding to a defined business segment or area of responsibility.

- Business Activity - unit of work that consumes and produces products. 
- Product - item that is produced of consumed during business activities. One or more roles develop a product in the performance of one or more activities.

- Product Kind - represents several types of products. Work products can be classified in several types, which identify the kind of input or/and output expected in an activity.

- Contract - formal or informal specification of agreement that specifies the rights and obligations associated with a business product.

- Business Role - responsibility for performing specific activities in order to produce, either directly or indirectly, versions of one or more products.

- Actor - organizational entity that performs one or more business roles.

Enriching business process meta-model with work practice information results in the action layer. In order to build a work practice Meta-model (WPM); there is a need for identifying the corresponding work practices constructs before the alignment with business process constructors. Figure 5 proposes an extension to the meta-model concerning work practice expressed in terms of entities and the relationships among them. The proposal conveys the following ideas:

- The entities individual, dyad and group that can be regarded both as actor or resources (of other actors).

- As actors, they perform several actions that use different kinds of resources (including other individuals, dyads or groups).

- Actions are not strictly classified into tasks, projects, etc. Rather, action streams are grouped in personal contexts.

- A single individual handles several personal contexts. At any given moment, individuals use personal scheduling rules to choose the context to work in. Likewise, two individuals (dyad) and groups activate inter-personal and group contexts using shared scheduling rules.

- An inter-personal context relates two personal contexts of two different individuals. Hence, the same two individuals (a unique dyad) may share several inter-personal contexts.

- Personal and inter-personal contexts may be related to one or several tasks/projects. Conversely, tasks/projects may be associated with several contexts.

- Actions create, update or delete resource-related items. These items may be related to one or more formal resources. Conversely, several items may compose a formal resource. The association of items with formal resources is user-defined.

- Communicative and non-communicative actions must be distinguished. The relationship of communicative actions with the obligations (to-dos) and commitments created, updated or cancelled by them, need to be provided.

- The notion of a person's state is included. This state is described in terms of the set of actions to-do and shared commitments. Knowing the person' state allows defining scheduling rules based on current commitments and actions to-do.

- Currently, the identification of an individual current context is based on the actions performed and resources used. This identification can be greatly enhanced if personal, inter-personal and group-level scheduling rules taking into account the individual state were known. 
The action layer of our meta-model describes a set of constructors to represent the relevant issues of organizational daily work practices. By instantiating the entities of the meta-model we are able to identify and map work practice constructors to business process constructors. This facilitates analysis and decision-making using views of different actors (individual, dyad and group in the action layer) to describe the actual business process of an organization (structure layer). The alignment between aspects of the action layers and its related business process aspects (Figure 6) is motivated by two considerations. First, daily actions change over time, and second, that change can be problematic since increases the gap with actual business process descriptions. This continued alignment is crucial for the capacity to manage change.

At the action layer, context is regarded as a group of related actions (personal action and interaction). At this level, personal action context reflects the personal view that the individual has of a given interaction context. The interaction context captures typical interactions between any two individuals. Whereas any two individuals share a single inter-personal relationship, they may share several interpersonal contexts. The inter-personal context represents interaction rules shared by two individuals, which governs the interactions patterns among them. The relationship of these patterns with business activities in the structure layer needs to be established through the identification and analysis of such contexts.

Figure 6

Alignment between Action and Structure Layers of the BPPAMeta-model

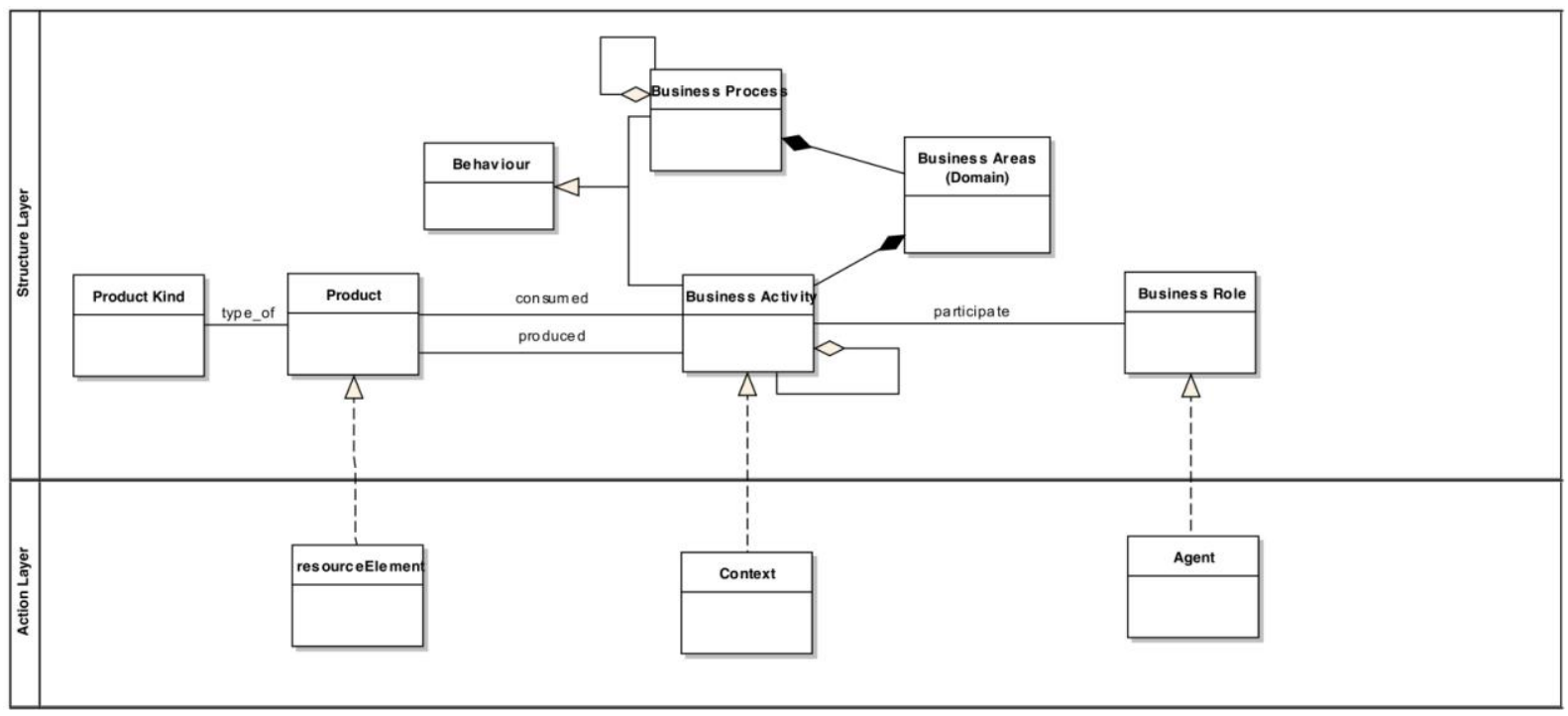

Source: Author's illustration

At the action layer, each interaction type is related to a specific set of resources types that enable, but also constrain, agent interactions. This relationship is essential to identify and associate products that consumed and produced by business activities in the structure layer. Each agent involved in the execution of specific actions must present a set of abilities and obligations. This means that is possible to infer specific roles (structure layer) of a business process based on agent abilities.

The dependencies of the constructors along these two layers (action layer and structure layer) form the structural backbone of the meta-model (BPPAMeta-model).

Contexts are formed by conversations (sequence of actions and interactions) among people around topics that may be related to one or several activities. The 
nature and structure of the conversation is determined by the activities involved and the role each person plays in them. Since people may interleave different topics of different activities within a conversation, it is not straight forward to associate a given context to a particular activity.

\section{Results and Discussion}

The meta-modeled proposed was applied in a case study. This section summarizes results obtained regarding the alignment of business process specifications with work practices. In this case contexts were analyzed to identify recurrent interaction patterns. Such analysis allowed identifying how different groups executed business activities and in what measure they deviated from pre-defined process specifications. Some interaction context features are illustrated in the following example, which is taken from a real organizational setting of a post-graduate educational institution. Consider the following sequence of actions:

- Prof. Smith request Alice the payment of a course he has lectured

- Alice check Prof. Smith' payment requirements (course grades and report) and notices it lacks the course report

- Alice request Prof. Smith to send the course grades and corresponding report

- Alice inform Prof. Smith that payment can only be made after receiving the required documentation

- Prof. Smith inform Alice that due to personal reasons, he can only send the documentation on date $D$

- Prof. Smith request an exception asking for the payment to be made prior to date D

- Alice analyzes Prof. Smith' request

- Alice asks her boss whether to accept Prof Smith's request

- Alice' boss answer that she should accept Prof. Smith's request because he has a very good record and consequently, deserves the exception requested

- Alice accepts Prof. Smith' request

- Alice orders Luisa the corresponding payment

- Alice informs Prof. Smith that payment is ordered

The previous sequence of actions created a context depicted in Figure 7. Such context is identified as 'Prof. Smith's payment situation' context. This context has three participants (agents): Alice, Prof. Smith and Alice's boss. The context reflects an agent network and its boundaries are defined first, by the three participating agents: Alice, Prof. Smith and Alice's boss. The topic 'Prof. Smith's payment situation' is the second criteria that help defining this context. The third and final criteria, is a set action types (request, analyze, inform, acknowledge, order, check, accept), and resources (telephone, mail, payment, requirements, knowledge about prof. Smith). The former and latter criteria can be used to uncover action and interaction patterns. Thereafter, identifying contexts means clustering actions within action repositories sharing a set of common features

\section{Contextual patterns}

Action repositories describe not only action types and its participants, they include descriptions of the information items, tools, materials or knowledge used or produced by each action. All this information allows finding detailed and personalized action and interaction patterns. Once found, action and interaction patterns can then be analyzed in order to identify to which business activity (and its encompassing business process) they belong to. It also allows identifying the role 
played by each agent in such activity. Patterns are also linked with formally defined resources already associated to given business activities or processes.

Figure 7

'Prof. Smith's payment situation' context

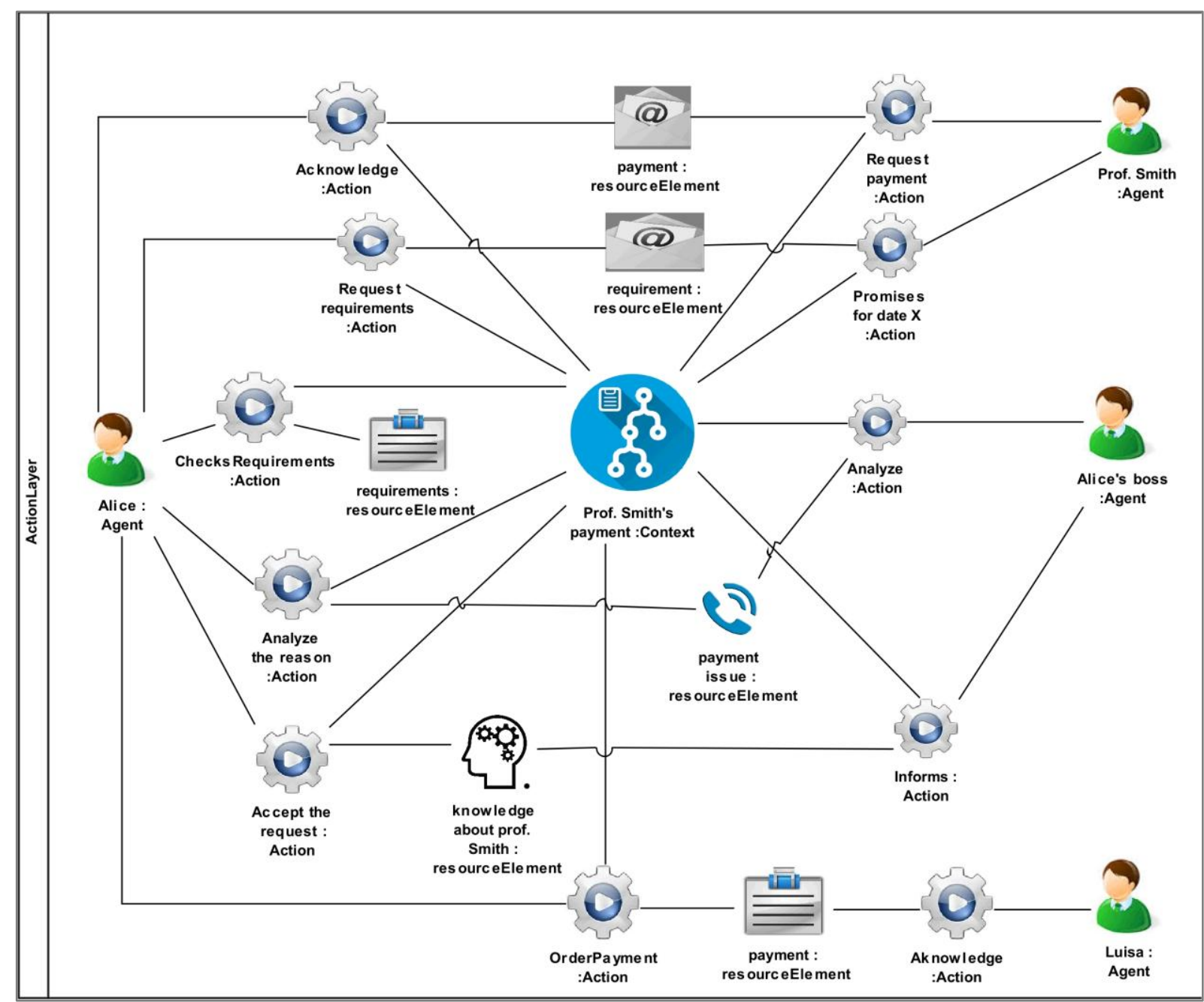

Source: Author's illustration

This bottom-up approach of linking work practices to business processes allows assessing deviations. Depending on their outcome, such deviations can be regarded as anomalies or innovations. After collecting work practice diagrams, the team discussed them and selected the ones they considered as best practices. Those best practices were then used to assess an existing business process. Figure 8 depicts a business process model that emerged from this discussion. In this case, the resulting business process reflects the course payment practice depicted in Figure 8 as it was considered a good practice by all teams. The previous conversation creates a context that is related to the Pay Course business activity (Figure 8). This context has two agents that play payer (Alice) and payee business roles (prof. Smith). Some of these actions will appear in the formal description of the activity Pay Course (request payment) but some will not (informing that the report will be sent a later date). Some resources are formal activity products (course grades and reports), and some will not due to their transient and informal nature (information that the report will be sent a later date and reason for the delay). 
Figure 8

'Pay course' business process (structure layer model)

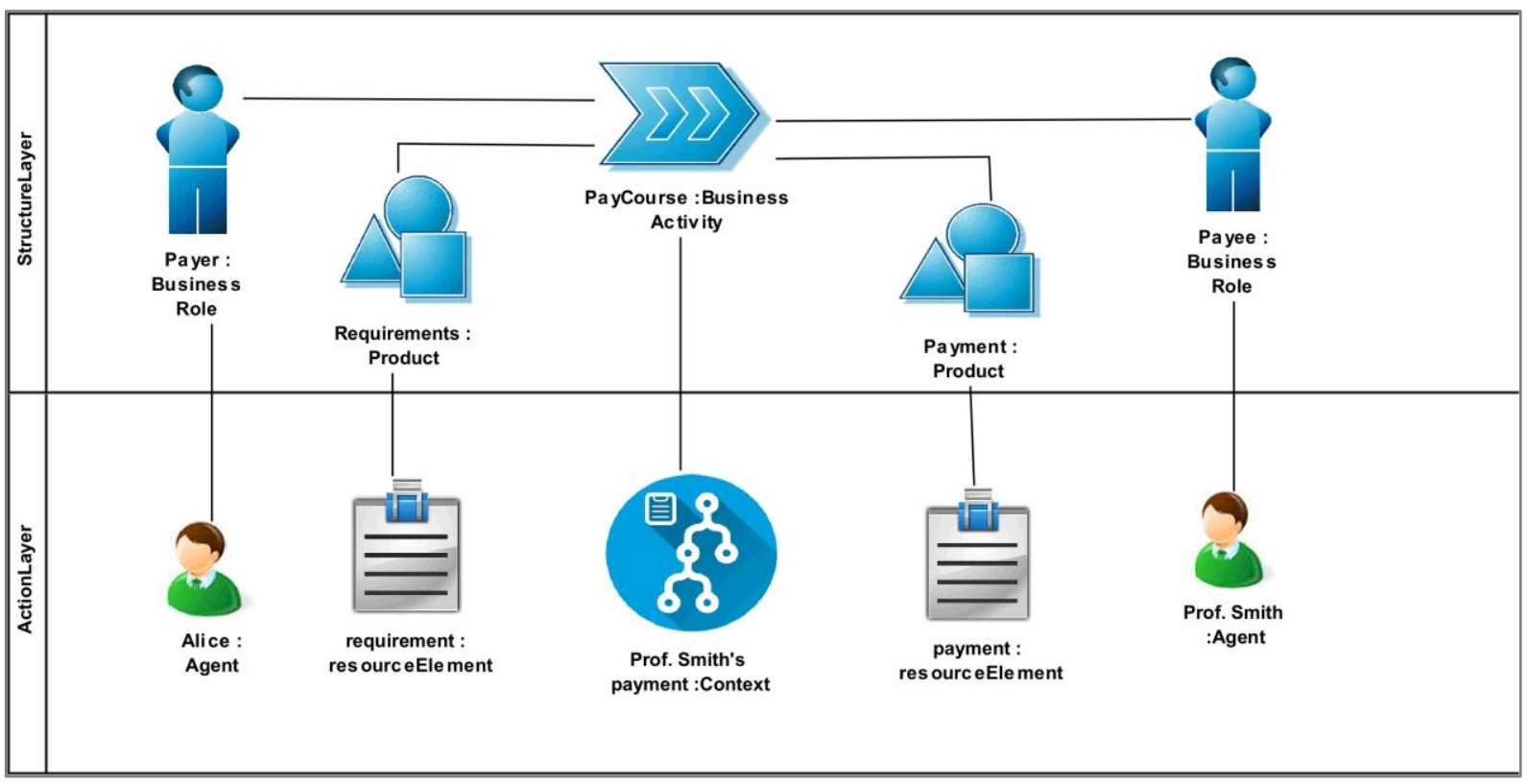

Source: Author's illustration

\section{Conclusion}

The different meta-models presented in related work focus different perspectives concerning with business process. The review of these meta-models allowed identifying the advantages and limits of each approach, each one concentrated on different aspects. The BPMN Meta-model belongs to the most well-known approaches that is used to create orchestrations of business processes internal to a specific organization and allows the definition of choreographies by interconnecting different processes. The QOBPM approach provides an integration of quality information to the corresponding business process constructs because without this quality data it is not possible to assess a business process. The TMBP approach contributes with support between organizational structure aspects and specific business process constructors as well as a catalogue of patterns based on different business process types. This study showed that although exist several business process meta-models, little effort has been devoted to the development of meta-models supporting the alignment between daily actions and business process descriptions as they are really executed in organizations.

In this paper, the BPPAMeta-model has been presented with its extension to integrate work practice information. The structure layer of the BPPAMeta-model allows designing functional, informational and behavioural aspects of business processes. The action layer extends the meta-model with work practice concepts that allow designing several aspects of daily actions. Moreover, structure and action layers had been aligned to perform the definitions of process elements based on work practice aspects. Besides, this meta-model can be used by the methodology BPPAM to specify or improve business processes models based on work practice descriptions. 


\section{References}

1. Bider, I., Jalali, A. (2016), "Agile business process development: why, how and when-applying Nonaka's theory of knowledge transformation to business process development", Information Systems and e-Business Management, Vol. 14 No. 4, pp. 693-731.

2. Castela, N., Dias, P., Zacarias, M., Tribolet, J. (2012), "Collaborative maintenance of business process models", International Journal of Organisational Design and Engineering, Vol. 2 No. 1, pp. 61-84.

3. Degani, A., Wiener, E. L. (1997), "Procedures in complex systems: the airline cockpit", IEEE Transactions on Systems, Man, and Cybernetics, Part A: Systems and Human, Vol. 27 No. 3, pp. 302-312.

4. Heidari, F., Loucopoulos, P., Kedad, Z. (2011), "A Quality-Oriented Business Process Meta-Model", 7th International Workshop, EOMAS 2011, held at CAiSE 2011, Springer Berlin Heidelberg, London.

5. Hevner, A., March, S., Park, J., Ram, S. (2004), "Design Science in Information Systems Research", MIS Quarterly, Vol. 28 No. 1, pp. 75-105.

6. Hollingaworth, D. (2004), "The Workflow Reference Model: 10 Years On", in Fischer, L. (Ed.), Workflow Handboook 2004, Future Strategies Inc., FL USA, pp. 295312.

7. Mutschler, B., Weber, B., Reichert, M. (2008), "Workflow management versus case handling: results from a controlled software experiment", Proceedings of the 2008 ACM symposium on Applied computing, ACM, Fortaleza, Ceara, Brazil, pp. 82-89.

8. OMG (2013), "Business Process Model and Notation (BPMN)", available at: http://www.omg.org/spec/BPMN/2.0.2/PDF/ (20 April 2017).

9. Pomerol, J. C., Brézillon, P. (2011), "About some relationships between knowledge and context", CONTEXT '01 Proceedings of the Third International and Interdisciplinary Conference on Modeling and Using Context, Springer-Verlag, London.

10. Reichert, M., Dadam, P., Jurisch, M., Kreher, U., Goser, K. (2008), "Architectural Design of Flexible Process Management Technology", PRIMIUM Subconference at the Multikonferenz Wirtschaftsinformatik (MKWI), Garching, Germany.

11. Sierhuis, M., Clancey, W. J., Hoof, R. V., Hoog, R. D. (2000), "Modeling and simulating work practices from Apollo 12", available at: https://pdfs.semanticscholar.org/3d76/f89964ab7a3cce44f3b7b543700350408afc .pdf (20 April 2017).

12. Sierhuis, M., Clancey, W. J. (1997), "Knowledge, practice, activities and people", available

at: https://pdfs.semanticscholar.org/0ad9/feccef60f8331727fd53a9eb34a15eb00f2c. pdf (20 April 2017).

13. Thom, L. H., lochpe, C., Mitschang, B. (2005), "TMBP: A Transactional Metamodel for Business Process Modeling Based on Organizational Structure Aspects", CAiSE Short Paper Proceedings, Porto.

14. Verner, L. (2004), "BPM: The Promise and the Challenge", Queve, Vol. 2 No. 1, pp. 82-91.

15. Zacarias, M., Magalhães, R., Pinto, H. S., Tribolet, J. (2010), "An agent-centric and 'context-aware' perspective for the alignment between individuals and organizations", Information Systems Journal, Vol. 35 No. 4, pp. 441-466.

16. Zacarias, M., Martins, P. V. (2014), "Business Alignment Methodology: The Discovery Phase", Information Resources Management Journal, Vol. 27 No. 1, pp. 1-20. 


\section{About the authors}

Paula Ventura Martins is an assistant professor of the Electronics and Computers Engineering Department of Faculty of Sciences and Technology, University of Algarve, and a member of Research Centre for Spatial and Organizational Dynamics (CIEO). She has a PhD degree in Computer Science and Engineering from the "Instituto Superior Técnico da Universidade Técnica de Lisboa" (IST/ULT). In 2000, she got the Master's degree in Computer Science and Engineering from the "Faculdade de Ciências e Tecnologia da Universidade Nova de Lisboa" (FCT/UNL). She concluded her undergraduate course in Computer Science and Engineering on 1992, in the same institution. Her personal interests are Software Process Improvement, Software Development Processes, Domain Specific Languages, Modelling Languages and Business Process Modelling. Author can be contacted at pventura@ualg.pt

Marielba Zacarias is a Computer Engineering (1982) with a MSc degree in Systems Engineering (1996) from "Universidad Simón Bolívar de Venezuela" (USB), and a PhD in Informatics and Computers Engineering (2008) from "Instituto Superior Técnico da Universidade Técnica de Lisboa" (IST/ULT). She has a professional experience of over 25 years in Information Systems. From 1983 to 2001, she first worked as software developer and later as a software project manager. In 2001, she moved to Portugal where she is dedicated exclusively to teaching and research activities. Currently, she is an assistant professor of the Electronics and Computers Engineering Department of Faculty of Sciences and Technology, University of Algarve, and a member of Research Centre for Spatial and Organizational Dynamics (CIEO). After her PhD, she has worked mainly in organizational modeling and ontologies, and has published several scientific papers in those areas. Author can be contacted at mzacaria@ualg.pt 